$04,09,11$

\title{
Электронная структура, оптические свойства и поведение под давлением в соединениях $\mathrm{CdB}_{4} \mathrm{O}_{7}$ и $\mathrm{HgB}_{4} \mathrm{O}_{7}$
}

\author{
(C) А.С. Шинкоренко, В.И. Зиненко, М.С. Павловский \\ Институт физики им. Л.В. Киренского СО РАН (ФИЦ КНЦ СО РАН), \\ Красноярск, Россия \\ E-mail: shas@iph.krasn.ru
}

(Поступила в Редакцию 15 марта 2018 г.)

В рамках теории функционала плотности с использованием пакета VASP из первых принципов выполнены расчеты структурных, электронных и оптических свойств соединений тетраборатов $\mathrm{HgB}_{4} \mathrm{O}_{7}$ и $\mathrm{CdB}_{4} \mathrm{O}_{7}$ в трех структурных модификациях с симметрией $\mathrm{Pbca}, \mathrm{Cmcm}$ и $\mathrm{Pmn} 2_{1}$. Расчеты электронной зонной структуры показали, что исследуемые соединения во всех рассматриваемых модификациях являются диэлектриками с шириной запрещенной зоны $2-4 \mathrm{eV}$. Расчеты структурных свойств исследуемых тетраборатов под давлением показали, что фазовый переход между структурами $P b c a$ и $P m n 2_{1}$ в тетраборате кадмия и тетраборате ртути происходит при давлениях $4.8 \mathrm{GPa}$ и $4.7 \mathrm{GPa}$ соответственно.

Работа выполнена при финансовой поддержке гранта РФФИ 16-32-00373 мол_а и с использованием оборудования Центра коллективного пользования „Комплекс моделирования и обработки данных исследовательских установок мега-класса“ НИЦ „Курчатовский институт“, http://ckp.nrcki.ru/.

DOI: 10.21883/FTT.2018.09.46381.069

\section{1. Введение}

Соединения боратов с химической формулой $\mathrm{M}^{+2} \mathrm{~B}_{4} \mathrm{O}_{7}$ обладают рядом интересных особенностей, таких как люминесценция, нелинейные оптические и электрические свойства [1]. Эти свойства могут иметь прикладное значение. В дополнение к этому они интересны с кристалохимической точки зрения. Данные соединения реализуются в нескольких основных структурных формах, содержащих $\mathrm{BO}_{3}$ - треугольники и/или $\mathrm{BO}_{4}$ - тетраэдры. Как показывают эксперименты [2-10], в зависимости от радиуса иона $\mathrm{M}^{+2}$ и внешних условий соединения $\mathrm{M}^{+2} \mathrm{~B}_{4} \mathrm{O}_{7}$ могут существовать в двух из трех основных структурных типов, за некоторыми исключениями [11].

Первый структурный тип принадлежит пространственной группе симметрии Pbca. Основу структуры этого типа составляет борокислородный каркас, состоящий из соединенных между собой вершинами тетраэдров $\mathrm{BO}_{4}$ и треугольников $\mathrm{BO}_{3}$ (в соотношении один к одному). В полостях такого каркаса располагаются атомы $\mathrm{M}^{+2}$. Этот структурный тип, как правило, реализуется в соединениях, в которых ионный радиус металла имеет величину в интервале $0.9 \AA-1 \AA$. Для соединений с ионным радиусом металла меньшем $1 \AA$ при высоких температурах и давлениях реализуется второй структурный тип с группой симметрии $\mathrm{Cmcm}$. В отличие от структуры с пространственной группой $P b c a$, в этой структуре присутствуют только борокислородные тетраэдры - $\mathrm{BO}_{4}$. И, наконец, третий структурный тип имеет полярную группу $P m n 2_{1}$, также состоит только из тетраэдров $-\mathrm{BO}_{4}$ и этот структурный тип при нормальном давлении реализуется в соединениях с радиусом иона металла большем $1.3 \AA$. Для соединений с радиусом иона металла в интервале $1.1 \AA<1.3 \AA$ этот структурный тип реализуется при высоких давлениях. Ионы кадмия и ртути имеют радиус иона $\sim 1.15 \AA$ и можно ожидать, что в тетраборатах с этими ионами могут реализоваться все три структурных типа.

Для тетрабората ртути известны как фаза нормального [12], так и фаза высокого давления [9]. Для соединения тетрабората кадмия экспериментально известна лишь фаза нормального давления [13]. В [14] авторы в рамках первопринципного расчета исследовали фазовую диаграмму $\mathrm{CdB}_{4} \mathrm{O}_{7}$ при гидростатическом давлении. Согласно их расчетам, в тетраборате кадмия под давлением $5 \mathrm{GPa}$ происходит фазовый переход из $\mathrm{Pbca}$ в $\mathrm{Cmcm}$. Исследований по определению электронных и оптических свойств данных соединений в литературе не найдено.

В данной статье представлены результаты первопринципных вычислений структурных, электронных и оптических свойств тетраборатов кадмия и ртути в фазах со структурами $P b c a, C m c m, P m n 2_{1}$ и исследуется их фазовая диаграмма под давлением.

\section{2. Метод расчета}

Расчеты проводились с использованием Projector Augmented Wave (PAW) [15,16] в DFT, реализованного в коде „The Vienna Ab initio Simulation Package“ (VASP) [17,18]. Использовалось обобщенное градиентное приближение GGA c параметризацией PerdewBurke-Ernzerhof [19]. Были выбраны электронные конфигурации для потенциалов атомов: $\mathrm{Cd}: 4 d^{10} 5 s^{2}, \mathrm{Hg}$ : $5 d^{10} 6 s^{2}$, В: $2 s^{2} 2 p^{1}$, О: $2 s^{2} 2 p^{4}$. Число плоских волн ограничивалось энергией $600 \mathrm{eV}$. Размер сетки $k$-точек на 
основе схемы Monkhorst-Pack [20] составлял $4 \times 5 \times 3$ (для типа структуры Pbca), $4 \times 6 \times 8$ (для структурного типа $C m c m$ ) и $4 \times 9 \times 9$ (для типа структуры $P m n 2_{1}$ ). Для вычисления DOS сетка $k$-точек увеличивалась в два раза в каждом направлении. Геометрия структур была оптимизирована до тех пор, пока остаточные силы не станут меньше $1 \mathrm{meV} / \AA$.

Расчеты электронной структуры и оптических свойств проводились на релаксированных параметрах решетки и координатах.

\section{3. Электронная структура}

В табл. 1-3 приведены вычисленные параметры ячейки и относительные координаты атомов для трех структурных модификаций соединений $\mathrm{CdB}_{4} \mathrm{O}_{7}$ и $\mathrm{HgB}_{4} \mathrm{O}_{7}$. Там же приведены имеющиеся в литературе экспе-

Таблица 1. Вычисленные и экспериментальные (в скобках) координаты атомов и параметры решетки соединений $\mathrm{CdB}_{4} \mathrm{O}_{7}[13]$ и $\mathrm{HgB}_{4} \mathrm{O}_{7}[12]$ в структуре $\mathrm{Pbca}(\mathrm{Z}=8)$

\begin{tabular}{c|c|c|c}
\hline$P b c a$ & $a$ & $b$ & $c$ \\
\hline $\mathrm{CdB}_{4} \mathrm{O}_{7}$ & $8.41(8.21) \AA$ & $8.90(8.70) \AA$ & $14.34(14.18) \AA$ \\
$\mathrm{HgB}_{4} \mathrm{O}_{7}$ & $8.548(8.399) \AA$ & $8.955(8.806) \AA$ & $14.371(14.137) \AA$ \\
\hline $\mathrm{M}^{\mathrm{Cd}}$ & $0.1262(0.1210)$ & $0.1105(0.1105)$ & $0.6138(0.6125)$ \\
$\mathrm{M}^{\mathrm{Hg}}$ & $0.1393(0.1394)$ & $0.1157(0.1122)$ & $0.1174(0.1169)$ \\
\hline $\mathrm{B}_{1}^{\mathrm{Cd}}$ & $0.4453(0.4473)$ & $0.2830(0.2864)$ & $0.5647(0.5679)$ \\
$\mathrm{B}_{1}^{\mathrm{Hg}}$ & $0.4516(0.4542)$ & $0.2876(0.2810)$ & $0.0623(0.0616)$ \\
\hline $\mathrm{B}_{2}^{\mathrm{Cd}}$ & $0.0156(0.0140)$ & $0.3218(0.3385)$ & $0.7658(0.7654)$ \\
$\mathrm{B}_{2}^{\mathrm{Hg}}$ & $0.5076(0.5109)$ & $0.3343(0.3294)$ & $0.2336(0.2348)$ \\
\hline $\mathrm{B}_{3}^{\mathrm{Cd}}$ & $0.0091(0.0061)$ & $0.0684(0.0640)$ & $0.8376(0.8376)$ \\
$\mathrm{B}_{3}^{\mathrm{Hg}}$ & $0.5188(0.5165)$ & $0.0699(0.0632)$ & $0.1618(0.1609)$ \\
\hline $\mathrm{B}_{4}^{\mathrm{Cd}}$ & $0.2048(0.2019)$ & $0.1476(0.1374)$ & $0.9549(0.9575)$ \\
$\mathrm{B}_{4}^{\mathrm{Hg}}$ & $0.7152(0.7176)$ & $0.1557(0.1487)$ & $0.0490(0.0489)$ \\
\hline $\mathrm{O}_{1}^{\mathrm{Cd}}$ & $0.0479(0.0543)$ & $0.4225(0.4103)$ & $0.6920(0.6875)$ \\
$\mathrm{O}_{1}^{\mathrm{Hg}}$ & $0.0337(0.0426)$ & $0.4246(0.4173)$ & $0.1924(0.1895)$ \\
\hline $\mathrm{O}_{2}^{\mathrm{Cd}}$ & $0.3890(0.3853)$ & $0.1387(0.1325)$ & $0.5994(0.5980)$ \\
$\mathrm{O}_{2}^{\mathrm{Hg}}$ & $0.4030(0.4003)$ & $0.1346(0.1332)$ & $0.0951(0.0933)$ \\
\hline $\mathrm{O}_{3}^{\mathrm{Cd}}$ & $0.3489(0.3544)$ & $0.3691(0.3759)$ & $0.4996(0.5021)$ \\
$\mathrm{O}_{3}^{\mathrm{Hg}}$ & $0.3574(0.3627)$ & $0.3584(0.3646)$ & $-0.0059(0.0064)$ \\
\hline $\mathrm{O}_{4}^{\mathrm{Cd}}$ & $0.4693(0.4733)$ & $0.3856(0.3837)$ & $0.6477(0.6469)$ \\
$\mathrm{O}_{4}^{\mathrm{Hg}}$ & $0.4611(0.4651)$ & $0.3845(0.3834)$ & $0.1467(0.1474)$ \\
\hline $\mathrm{O}_{5}^{\mathrm{Cd}}$ & $0.0322(0.0335)$ & $0.1773(0.1642)$ & $0.7567(0.7545)$ \\
$\mathrm{O}_{5}^{\mathrm{Hg}}$ & $0.5300(0.5288)$ & $0.1810(0.1730)$ & $0.2440(0.2448)$ \\
\hline $\mathrm{O}_{6}^{\mathrm{Cd}}$ & $0.1016(0.1094)$ & $0.2611(0.2561)$ & $0.9831(0.9799)$ \\
$\mathrm{O}_{6}^{\mathrm{Hg}}$ & $0.6120(0.6153)$ & $0.2679(0.2650)$ & $0.0210(0.0208)$ \\
\hline $\mathrm{O}_{7}^{\mathrm{Cd}}$ & $0.1634(0.1595)$ & $0.0546(0.0428)$ & $0.8853(0.8841)$ \\
$\mathrm{O}_{7}^{\mathrm{Hg}}$ & $0.6725(0.6733)$ & $0.0526(0.0617)$ & $0.1189(0.1180)$ \\
& & & \\
\hline
\end{tabular}

Таблица 2. Вычисленные координаты атомов и параметры решетки соединений $\mathrm{CdB}_{4} \mathrm{O}_{7}$ и $\mathrm{HgB}_{4} \mathrm{O}_{7}$ в структуре $\mathrm{Cmcm}$ $(Z=4)$

\begin{tabular}{c|c|c|c}
\hline$C m c m$ & $a$ & $b$ & $c$ \\
\hline $\mathrm{CdB}_{4} \mathrm{O}_{7}$ & $11.062 \AA$ & $6.838 \AA$ & $5.325 \AA$ \\
$\mathrm{HgB}_{4} \mathrm{O}_{7}$ & $11.118 \AA$ & $6.896 \AA$ & $5.383 \AA$ \\
\hline $\mathrm{M}^{\mathrm{Cd}}$ & 0.0000 & 0.2163 & 0.2500 \\
$\mathrm{M}^{\mathrm{Hg}}$ & 0.0000 & 0.2162 & 0.2500 \\
\hline $\mathrm{B}_{1}^{\mathrm{Cd}}$ & 0.7105 & 0.0000 & 0.0000 \\
$\mathrm{~B}_{1}^{\mathrm{Hg}}$ & 0.7123 & 0.0000 & 0.0000 \\
\hline $\mathrm{B}_{2}^{\mathrm{Cd}}$ & 0.6213 & 0.2886 & 0.2500 \\
$\mathrm{~B}_{2}^{\mathrm{Hg}}$ & 0.6222 & 0.2861 & 0.2500 \\
\hline $\mathrm{O}_{1}^{\mathrm{Cd}}$ & 0.0000 & 0.8851 & 0.2500 \\
$\mathrm{O}_{1}^{\mathrm{Hg}}$ & 0.0000 & 0.8776 & 0.2500 \\
\hline $\mathrm{O}_{2}^{\mathrm{Cd}}$ & 0.2872 & 0.4698 & 0.2500 \\
$\mathrm{O}_{2}^{\mathrm{Hg}}$ & 0.2882 & 0.4674 & 0.2500 \\
\hline $\mathrm{O}_{3}^{\mathrm{Cd}}$ & 0.1380 & 0.3267 & -0.0285 \\
$\mathrm{O}_{3}^{\mathrm{Hg}}$ & 0.1407 & 0.3285 & -0.0317
\end{tabular}

Таблица 3. Вычисленные и экспериментальные [11] координаты атомов и параметры решетки соединений $\mathrm{CdB}_{4} \mathrm{O}_{7}$ и $\mathrm{HgB}_{4} \mathrm{O}_{7}$ в структуре $P m n 2_{1}(Z=2)$

\begin{tabular}{c|c|c|c}
\hline$P m n 2_{1}$ & $a$ & $b$ & $c$ \\
\hline $\mathrm{CdB}_{4} \mathrm{O}_{7}$ & $10.769 \AA$ & $4.420 \AA$ & $4.226 \AA$ \\
$\mathrm{HgB}_{4} \mathrm{O}_{7}$ & $10.825(10.656) \AA$ & $4.445(4.381) \AA$ & $4.239(4.187) \AA$ \\
\hline $\mathrm{M}^{\mathrm{Cd}}$ & 0.0000 & 0.1648 & 0.1033 \\
$\mathrm{M}^{\mathrm{Hg}}$ & $0.0000(0.0000)$ & $0.1800(0.1802)$ & $0.1033(0.1033)$ \\
\hline $\mathrm{B}_{1}^{\mathrm{Cd}}$ & 0.8794 & 0.6721 & 0.6359 \\
$\mathrm{~B}_{1}^{\mathrm{Hg}}$ & $0.8794(0.8796)$ & $0.6768(0.6738)$ & $0.5869(0.5900)$ \\
\hline $\mathrm{B}_{2}^{\mathrm{Cd}}$ & 0.7510 & 0.8259 & 0.1596 \\
$\mathrm{~B}_{2}^{\mathrm{Hg}}$ & $0.7513(0.7513)$ & $0.8301(0.8258)$ & $0.0696(0.0704)$ \\
\hline $\mathrm{O}_{1}^{\mathrm{Cd}}$ & 0.7777 & 0.8644 & 0.8027 \\
$\mathrm{O}_{1}^{\mathrm{Hg}}$ & $0.7791(0.7762)$ & $0.8700(0.8637)$ & $0.4197(0.4272)$ \\
\hline $\mathrm{O}_{2}^{\mathrm{Cd}}$ & 0.8600 & 0.3530 & 0.7127 \\
$\mathrm{O}_{2}^{\mathrm{Hg}}$ & $0.8583(0.8615)$ & $0.3586(0.3518)$ & $0.5153(0.5155)$ \\
\hline $\mathrm{O}_{3}^{\mathrm{Cd}}$ & 0.8661 & 0.7316 & 0.2985 \\
$\mathrm{O}_{3}^{\mathrm{Hg}}$ & $0.8653(0.8667)$ & $0.7361(0.7275)$ & $0.9235(0.9322)$ \\
\hline $\mathrm{O}_{4}^{\mathrm{Cd}}$ & 0.000 & 0.7716 & 0.7456 \\
$\mathrm{O}_{4}^{\mathrm{Hg}}$ & $0.0000(0.0000)$ & $0.7701(0.7770)$ & $0.4787(0.4809)$
\end{tabular}

риментальные данные. Из приведенных таблиц видно хорошее согласие с экспериментальными данными как параметров ячейки, так и координат атомов.

Вычисленная электронная зонная структура соединений $\mathrm{CdB}_{4} \mathrm{O}_{7}$ и $\mathrm{HgB}_{4} \mathrm{O}_{7}$ в структуре с группой симметрии $P b c a$ показана на рис. 1. Качественных отличий в 

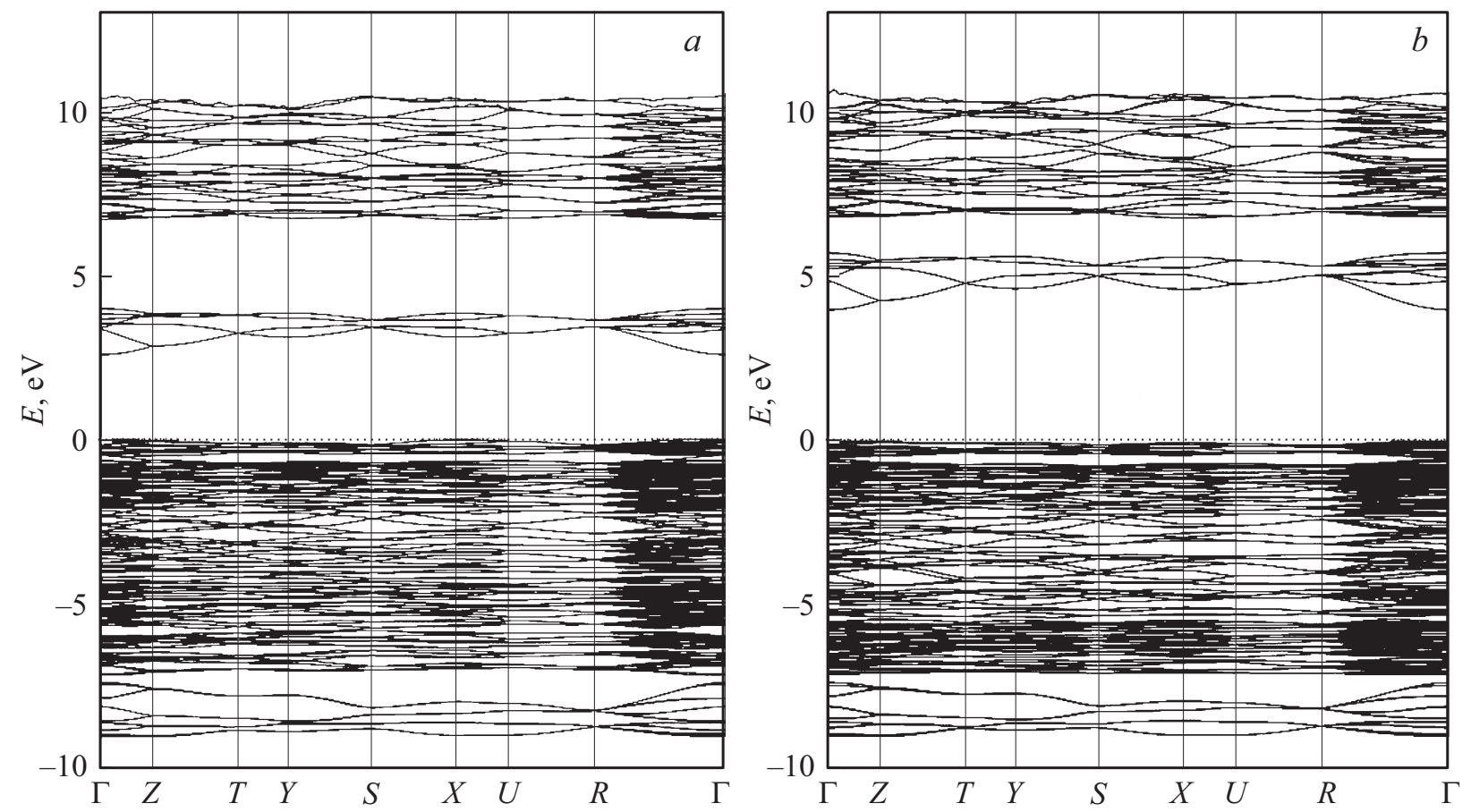

Рис. 1. Вычисленная электронная зонная структура соединений $\mathrm{HgB}_{4} \mathrm{O}_{7}(a)$ и $\mathrm{CdB}_{4} \mathrm{O}_{7}(b)$ в структуре $P b c a$.

электронной структуре этих соединений не обнаружено. Полная (TDOS) и частичная (PDOS) плотности состояний для рассматриваемых соединений в трех структурах приведены на рис. 2. Bce TDOS и PDOS нормированы на формульную единицу. Ширина запрещенной зоны $\Delta E$ в разных структурах исследуемых соединений приведена в табл. 4.

Вычисленные электронные TDOS и PDOS для различных структурных типов кристаллов $\mathrm{CdB}_{4} \mathrm{O}_{7}$ и $\mathrm{HgB}_{4} \mathrm{O}_{7}$ качественно не отличаются. Видно, что структура валентной зоны почти одинакова во всех модификациях рассматриваемых соединений. Она состоит из $d$-состояний атомов $\mathrm{M}^{2+}$ (середина зоны, высокий пик), $s$ и $p$-состояний атомов В (центр и дно зоны) и p-состояний атомов О (центр и верхняя часть зоны).

Наибольшие различия наблюдаются в зоне проводимости для всех типов структур. Во всех рассматриваемых случаях нижняя узкая часть зоны проводимости отделена от основной части (величина данного интервала энергий $\Delta E_{1}$ приведена в табл. 4). Эта нижняя часть

Таблица 4. Вычисленные значения ширины запрещенной зоны $\Delta E$ и $\Delta E_{1}$ соединений $\mathrm{CdB}_{4} \mathrm{O}_{7}$ и $\mathrm{HgB}_{4} \mathrm{O}_{7}$ в различных структурах

\begin{tabular}{c|c|c|c|c|c|c}
\hline & \multicolumn{3}{|c|}{$\mathrm{CdB}_{4} \mathrm{O}_{7}$} & \multicolumn{3}{c}{$\mathrm{HgB}_{4} \mathrm{O}_{7}$} \\
\cline { 2 - 7 } & Pbca & $C m c m$ & Pmn2 $_{1}$ & Pbca & Cmcm & Pmn2 \\
\hline$\Delta E, \mathrm{eV}$ & 3.9 & 4.6 & 3.1 & 2.57 & 3.0 & 1.9 \\
$\Delta E_{1}, \mathrm{eV}$ & 1.0 & 0.9 & 2.9 & 2.7 & 2.2 & 4.1
\end{tabular}

зоны проводимости состоит из $s$-состояний электронов атомов $\mathrm{M}^{2+}$ и p-состояний электронов атомов О. В зависимости от структуры и состава дно зоны проводимости изменяет свое положение относительно верхней части валентной зоны, что в свою очередь меняет ширину запрещенной зоны. Наибольшая ширина запрещенной зоны наблюдается в структуре с группой симметрии Cmcm. Наименьшая ширина зоны в структуре $P m n 2_{1}$. Верхняя часть зоны проводимости для всех типов структур и соединений состоит из $p$-состояний электронов каждого атома.

В тетраборате ртути во всех структурах ширина запрещенной зоны меньше, чем в тетраборате кадмия, так как возбужденные $s$-состояния электронов ионов $\mathrm{Hg}$ находятся на более низком энергетическом уровне, чем возбужденные состояния $s$-электронов ионов $\mathrm{Cd}$ [21]. Верхняя часть зоны проводимости в обоих соединениях расположена примерно на одном уровне энергии.

\section{4. Оптические свойства}

Для всех типов структур для $\mathrm{CdB}_{4} \mathrm{O}_{7}$ и $\mathrm{HgB}_{4} \mathrm{O}_{7}$ были вычислены диэлектрическая функция [22], и коэффициент поглощения $\alpha$ от частоты [23]:

$$
\alpha(\omega)=\frac{2 \omega}{c} \sqrt{\frac{|\varepsilon(\omega)|-\varepsilon^{\prime}(\omega)}{2}},
$$

где $\varepsilon(\omega)$ - комплексная диэлектрическая функция, $\varepsilon^{\prime}(\omega)$ - вещественная часть диэлектрической функции. Вычисленные зависимости коэффициента поглощения 

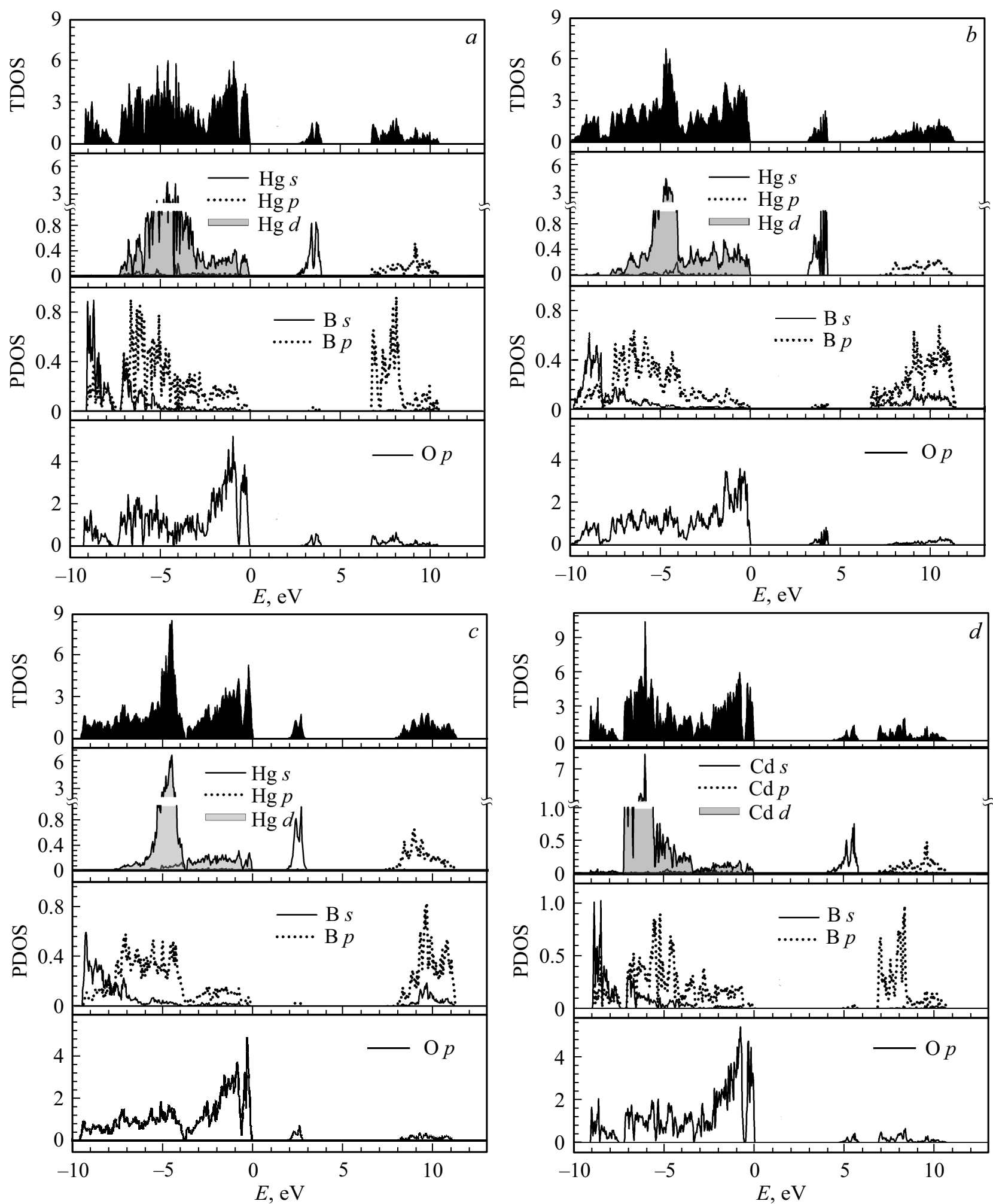

Рис. 2. Вычисленные TDOS и $\mathrm{PDOS}$ соединений $\mathrm{HgB}_{4} \mathrm{O}_{7}(a, b, c)$ и $\mathrm{CdB}_{4} \mathrm{O}_{7}(d, e, f)$ в различных структурах $P b c a$, Cmcm, Pmn $2_{1}$ соответственно.

показаны на рис. 3. Существенной анизотропии в оптических свойствах обнаружено не было, поэтому на рисунках приведено среднее значение коэффициента поглощения. Из этих зависимостей можно определить край поглощения материала соответственно для трех структур кристалла $\mathrm{CdB}_{4} \mathrm{O}_{7}:$ Pbca $\sim 300 \mathrm{~nm}, \mathrm{Cmcm} \sim 250 \mathrm{~nm}$, Pmn2 1 $350 \mathrm{~nm}$ и кристалла $\mathrm{HgB}_{4} \mathrm{O}_{7}:$ Р $C m c m \sim 370 \mathrm{~nm}, \mathrm{Pmn}_{1} \sim 600 \mathrm{~nm}$. Полученные значения 

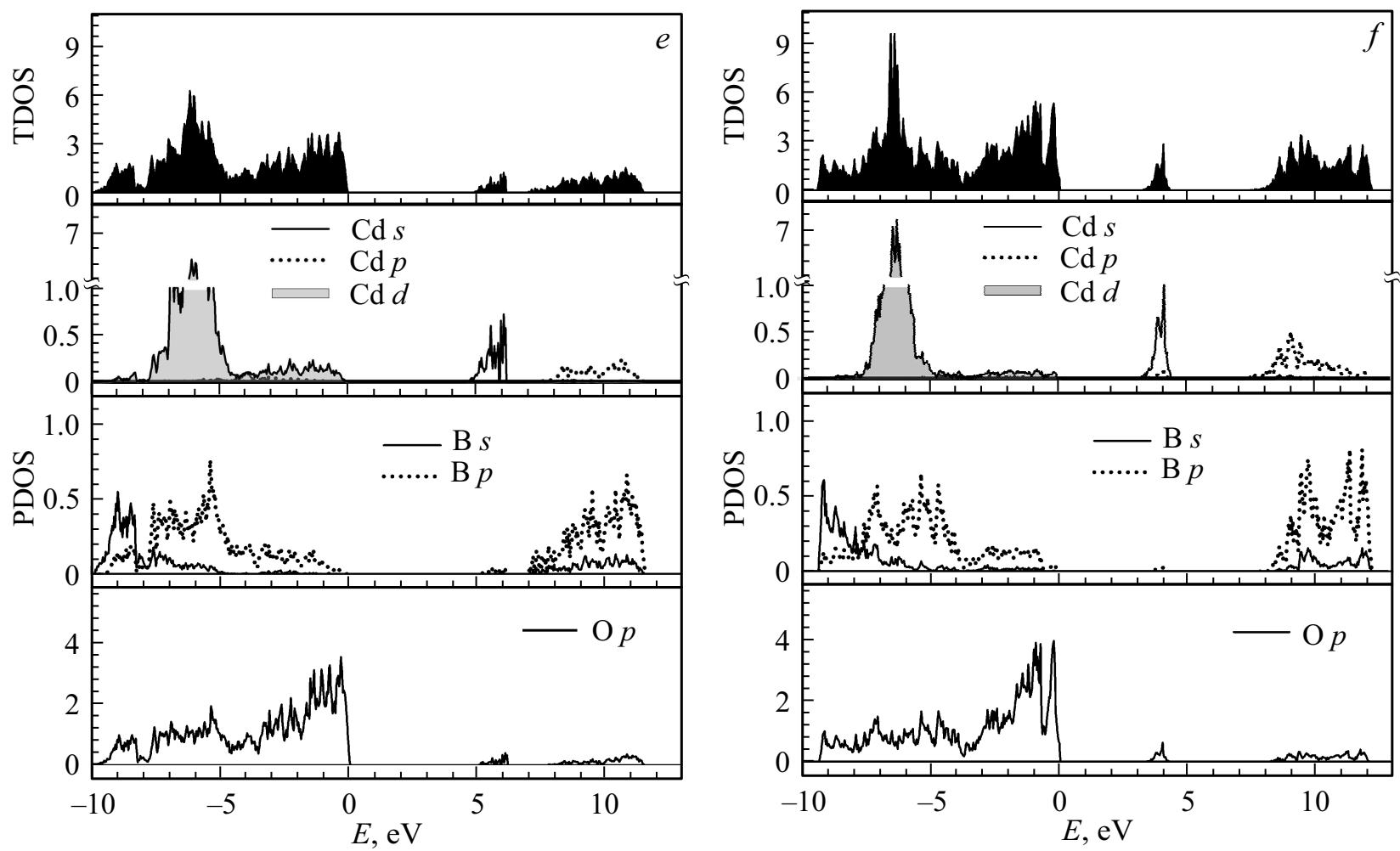

Рис. 2 (продолжение).
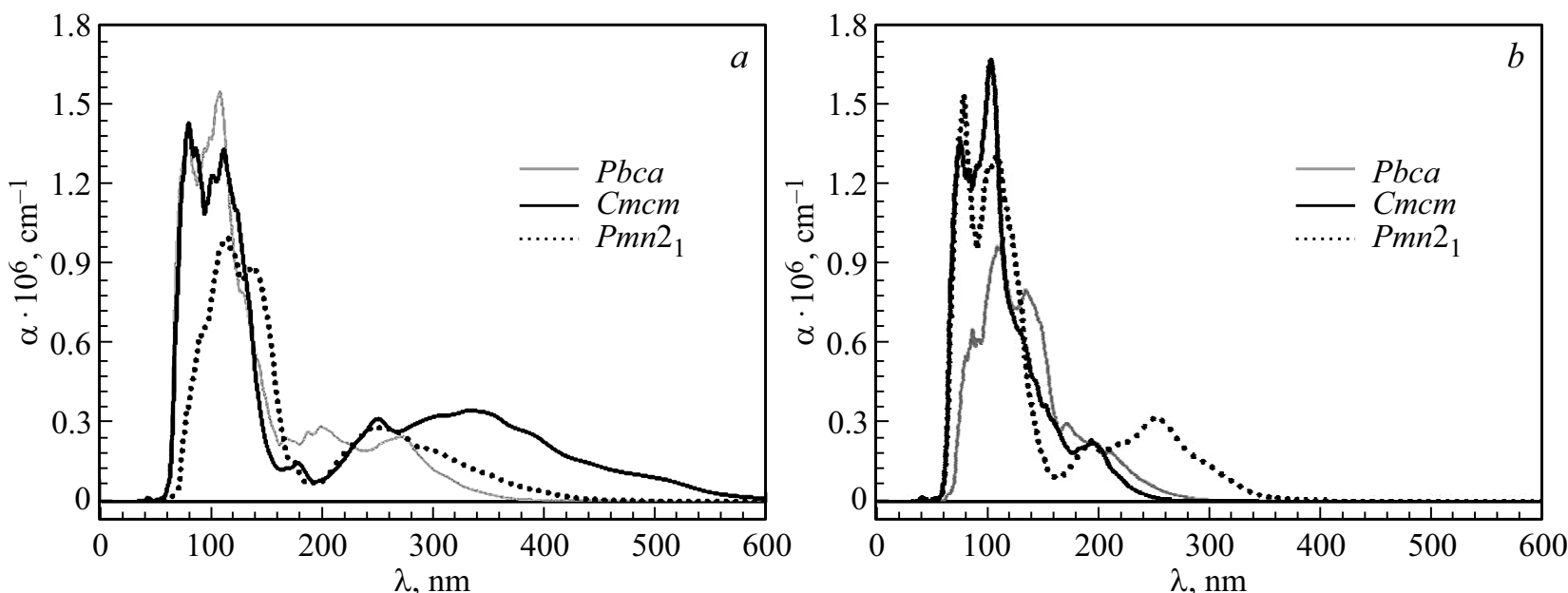

Рис. 3. Вычисленная зависимость коэффициента поглощения $\alpha$ от длины волны для соединений $\mathrm{HgB}_{4} \mathrm{O}_{7}(a)$ и $\mathrm{CdB}_{4} \mathrm{O}_{7}(b)$ в различных структурах.

края поглощения соответствуют особенностям вычисленных зонных структур, описанным выше.

\section{5. Упругие свойства и поведение под гидростатическим давлением}

Экспериментально установлено, что приложение гидростатического давления влияет на образование в структурах борокислородных тетраэдров $-\mathrm{BO}_{4}$ при росте кристаллов тераборатов двухвалентных металлов. В соединениях с малым ионным радиусом двухвалентного иона, как правило, конкурируют структуры с пространственными группами Pbca в Cmcm. Например, соединение $\mathrm{ZnB}_{4} \mathrm{O}_{7}$ при нормальном давлении кристаллизуется в пространственной группе Pbca, а при высоких давлениях $\mathrm{ZnB}_{4} \mathrm{O}_{7}$ кристаллизуется в пространственной группе $\mathrm{Cmcm}$ [8]. В соединениях с бо́льшим ионным радиусом конкурируют структуры с пространственными группами $\mathrm{Pbca}$ и $P m n 2_{1}$. Например, $\mathrm{SnB}_{4} \mathrm{O}_{7}$ при 

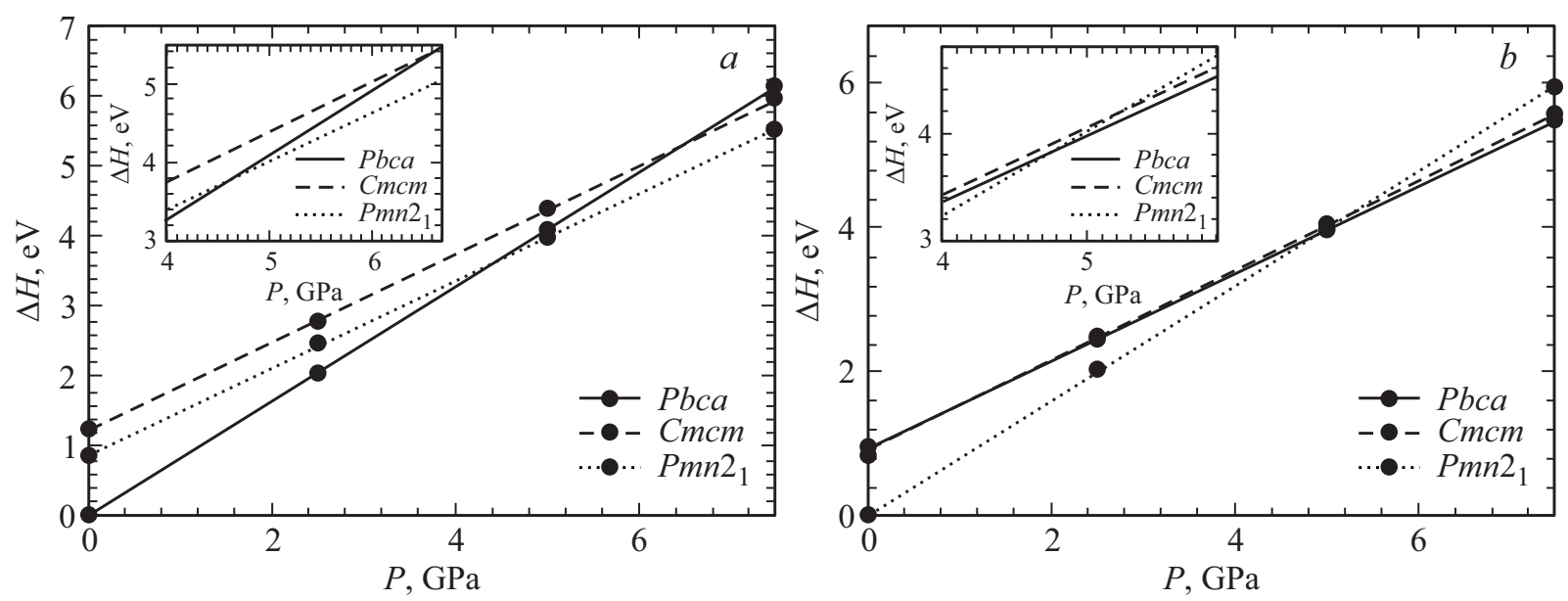

Рис. 4. Зависимость разницы энтальпии соединений $\mathrm{HgB}_{4} \mathrm{O}_{7}(a)$ и $\mathrm{CdB}_{4} \mathrm{O}_{7}(b)$ в трех структурах от давления. Точкой отсчета является энергия.

высоком давлении кристаллизуется в пространственной группе $P m n 2_{1}$. Структура фазы при нормальном давлении $\mathrm{SnB}_{4} \mathrm{O}_{7}$ не установлена [9]. Граничный радиус иона, где могут конкурировать все три рассматриваемые здесь структурных типа, составляет приблизительно $\sim 1.15 \AA$. Таким ионным радиусом обладают атомы $\mathrm{Cd}$ $\left(R_{\mathrm{Cd}}=1.15 \AA\right) \quad$ и $\mathrm{Hg} \quad\left(R_{\mathrm{Hg}}=1.14 \AA\right)$. Энергетическая выгодность фаз с пространственными группами $P m n 2_{1}$ и $\mathrm{Cmcm}$ (где, как уже отмечалось, присутствуют только $\mathrm{BO}_{4}$ тетраэдры) при высоких давлениях по сравнению с фазой с пространственной группой $\mathrm{Pbca}$ (где в равной пропорции присутствуют $\mathrm{BO}_{4}$ тетраэдры и $\mathrm{BO}_{3}$ треугольники) связана, по-видимому, с различием химических связей в этих фазах и, соответственно, с различием упругих свойств. В табл. 5 представлены вычисленные в данной работе значения упругих постоянных $\mathrm{CdB}_{4} \mathrm{O}_{7}$ и $\mathrm{HgB}_{4} \mathrm{O}_{7}$ в фазах с пространственными группами $P m n 2_{1}$, Cmcm и Рbca.

Как видно из табл. 5, упругие свойства рассматриваемых соединений в структурах $P m n 2_{1}$ и $\mathrm{Cmcm}$, содержащих только борокислородные тетраэдры, существенно отличаются от упругих свойств этих соединений

Таблица 5. Вычисленные значения упругих постоянных $C_{i i}[\mathrm{GPa}]$ соединений $\mathrm{CdB}_{4} \mathrm{O}_{7}$ и $\mathrm{HgB}_{4} \mathrm{O}_{7}$ в различных структурах

\begin{tabular}{l|l|r|r|r|r|r|r|r|r|r}
\hline \multirow{2}{*}{$\mathrm{CdB}_{4} \mathrm{O}_{7}$} & & $C_{11}$ & $C_{22}$ & $C_{33}$ & $C_{12}$ & $C_{13}$ & $C_{23}$ & $C_{44}$ & $C_{55}$ & $C_{66}$ \\
\cline { 2 - 11 } & $P b c a$ & 87 & 85 & 89 & 17 & 34 & 39 & 20 & 32 & 50 \\
\cline { 2 - 11 } & $C m c m$ & 370 & 318 & 338 & 147 & 128 & 114 & 85 & 131 & 115 \\
\cline { 2 - 10 } & $P m n 2_{1}$ & 261 & 272 & 334 & 30 & 30 & 28 & 64 & 90 & 78 \\
\hline $\mathrm{HgB}_{4} \mathrm{O}_{7}$ & $P b c a$ & 93 & 91 & 66 & 16 & 24 & 38 & 15 & 30 & 47 \\
\cline { 2 - 10 } & $C m c m$ & 352 & 302 & 321 & 148 & 136 & 110 & 84 & 121 & 96 \\
\cline { 2 - 10 } & $P m n 2_{1}$ & 267 & 279 & 335 & 42 & 40 & 42 & 38 & 93 & 56
\end{tabular}

со структурой $P b c a$, состоящей из борокислородных треугольников и тетраэдров в равной пропорции.

Чтобы оценить давление, при котором происходят фазовые переходы в кристаллах $\mathrm{CdB}_{4} \mathrm{O}_{7}$ и $\mathrm{HgB}_{4} \mathrm{O}_{7}$, для каждой из фаз $\mathrm{Pbca}, \mathrm{Cmcm}$ и $P m n 2_{1}$ проводилась релаксация параметров ячейки и координат ионов при определенных значениях гидростатического давления. При каждом давлении сравнивались значения энтальпии $H=E+P V$ (где $E-$ полная энергия кристалла, $P-$ давление, $V$ - объем ячейки), приходящейся на одну формульную единицу. Результаты расчета приведены на рис. 4. Из рисунка видно, что фазовый переход из $P b c a$ в $P m n 2_{1}$ в тетраборате кадмия происходит при давлении 4.8 GPa. Тот же фазовый переход в тетраборате ртути происходит при давлении 4.7 GPa. Полученное значение давления, при котором происходит фазовый переход в тетраборате ртути, качественно согласуется с экспериментальными данными работы [9], где кристалл $\mathrm{HgB}_{4} \mathrm{O}_{7}$ в фазе $P m n 2_{1}$ был выращен при давлении $7.5 \mathrm{GPa}$. В настоящих расчетах фазовый переход из $\mathrm{Pbca}$ в $\mathrm{Cmcm}$ не наблюдается. Стоит отметить, что разница в энергии между фазами $C m c m$ и $P m n 2_{1}$ в соединении $\mathrm{CdB}_{4} \mathrm{O}_{7}$ во всем интервале используемых при расчете значений давления очень мала ( $\Delta E_{\mathrm{tot}}=0.05 \mathrm{eV} /$ per formula).

\section{6. Заключение}

В рамках теории функционала плотности с использованием пакета VASP выполнены расчеты структурных, электронных и оптических свойств тетраборатов ртути и кадмия в трех структурных модификациях с симметрией $\mathrm{Pbca}, \mathrm{Cmcm}$ и Pmn2 ${ }_{1}$. Расчет показал, что в обоих соединениях во всех рассмотренных структурах величина энергетической щели между валентной зоной и зоной проводимости составляет порядка $2-4 \mathrm{eV}$. Ocoбенностью электронной структуры данных соединений является то, что нижняя часть зоны проводимости представляет собой узкую зону из $s$-состояний электронов 
атомов металла и $p$-состояний электронов атомов кислорода и эта нижняя часть отделена от основной части зоны проводимости на величину порядка нескольких электрон-вольт.

В результате расчета получено, что упругие свойства исследуемых соединений в структурах $P m n 2_{1}$ и $\mathrm{Cmcm}$, состоящих из борокислородных тетраэдров, существенно отличаются от упругих свойств этих соединений со структурой $P b c a$, состоящей из борокислородных треугольников и тетраэдров в равной пропорции. Проведено исследование свойств тетраборатов кадмия и ртути под давлением в различных фазах. Расчеты свойств $\mathrm{HgB}_{4} \mathrm{O}_{7}$ и $\mathrm{CdB}_{4} \mathrm{O}_{7}$ под давлением показали, что фазовый переход между структурами Pbca и Pmn $2_{1}$ в тетраборате кадмия и тетраборате ртути происходит при давлении $4.8 \mathrm{GPa}$ и $4.7 \mathrm{GPa}$ соответственно. Установлено, что переход между структурами $C m c m$ и $P m n 2_{1}$ в рассматриваемом в рамках настоящего расчета интервале давлений (до $50 \mathrm{GPa}$ ) не наблюдается. Однако энергии фаз со структурами Pbca и Pmn2 $2_{1}$ в $\mathrm{CdB}_{4} \mathrm{O}_{7}$ близки $\left(\Delta E_{\text {tot }}=0.05 \mathrm{eV} /\right.$ per formula) во всем интервале используемых в расчете величин давления.

\section{Список литературы}

[1] P. Becker. Adv. Mater. 10, 13, 979 (1998).

[2] M. Martinez-Rippol, S. Martinez-Carrera, S. Garcia-Blanco. Acta Cryst. B 27, 672 (1971).

[3] R.D. Shannon. Acta Cryst. A 32, 751 (1976).

[4] M. Prokic. Nucl. Instrum. Meth. 175, 1, 83 (1980).

[5] J. Krogh-Moe. Acta Chem. Scand. 18, 2055 (1964).

[6] K. Machida, H. Hata, K. Okuno, G. Adachi, J. Shiokawa. J. Inorg. Nucl. Chem. 41, 1425 (1979).

[7] H. Huppertz. Z. Naturforsch. 58b, 257 (2003).

[8] H. Huppertz, G. Heymann. Solid State Sci. 5, 2, 281 (2003).

[9] J.S. Knyrim, F.M. Schappacher, R. Pottgen, J.S. auf der Günne, D. Johrendt, H. Huppertz. Chem. Mater. 19, 2, 254 (2007).

[10] H. Emmea, M. Weilb, H. Huppertz. Z. Naturforsch. 60b, 815 (2005).

[11] J. S. Knyrim, S. Romer, W. Schnick, H. Huppertz. Solid State Sci. 11, 2, 336 (2009).

[12] M. Weil. Acta Cryst. E 59, 40 (2003).

[13] M.Ihara, J. Krogh-Moe. Acta Cryst. 20, 132 (1966).

[14] B. Winkler, A.G. Castellanos Guzman, L. Wiehl, L. Bayarjargal, V. Milman. Solid State Sci. 14, 8, 1080 (2012).

[15] G. Kresse, D. Joubert. Phys. Rev. B 59, 1758 (1999).

[16] P.E. Blochl. Phys. Rev. B 50, 17953 (1994).

[17] G. Kresse, J. Hafner. Phys. Rev. B 47, 558 (1993).

[18] G. Kresse, J. Furthmuller. Phys. Rev. B 54, 11169 (1996).

[19] J.P. Perdew. In: Electronic Structures of Solids' 91 / Eds P. Ziesche, H. Eschrig. Akademie Verlag, Berlin (1991). P. 11.

[20] H.J. Monkhorst. J.D. Pack. Phys. Rev. B 13, 5188 (1976).

[21] J.A. Mc Leod, R.J. Green, N.A. Skorikov, L.D. Finkelstein, M. Abu-Samak, E.Z. Kurmaev, A. Moewes. Proc. SPIE 7940, 79400R-1 (2011).

[22] M. Gajdoš, K. Hummer, G. Kresse, J. Furthmüller, F. Bechstedt. Phys. Rev. B 73, 045112 (2006).

[23] F. Han. A Modern Course in The Quantum Theory of Solids. World Scientific Publishing Co. Pte. Ltd, Singapore (2012). $720 \mathrm{p}$.

Редактор Т.Н. Василевская 